\title{
Review of: "Asymptomatic SARS-CoV-2 infection: A systematic review and meta-analysis"
}

Petrick Periyasamy

Potential competing interests: The author(s) declared that no potential competing interests exist.

1. The search strategy, methodology was clearly laid out and well defined. Basically 3 databases was searched - Medline (Pubmed), Embase and WHO. WOS would not reveal much but at least 2 big database tick!

2. Meta-analysis was done. And there was a lot of work to analyse 350 research papers.

3. The writing was simple and the objective of the paper was actually captured

5. Bias assessment was done. 